\title{
ON AN ALGEBRAIC FORMULA AND APPLICATIONS TO GROUP ACTION ON MANIFOLDS*
}

\author{
PING LI $^{\dagger}$ AND KEFENG LIU
}

\begin{abstract}
In this paper we consider a purely algebraic result. Then given a circle or cyclic group of prime order action on a manifold, we will use it to estimate the lower bound of the number of fixed points. We also give an obstruction to the existence of $\mathbb{Z}_{p}$ action on manifolds with isolated fixed points when $p$ is a prime.
\end{abstract}

Key words. Group actions, fixed points, localization formulae.

AMS subject classifications. 19J35, 37B05.

1. Introduction. In this short note we first prove a purely algebraic result, which stems from the localization formulae of group actions on manifolds, and the idea of which has been used in several literatures ([8], [6], [5], [7], [2]). Then, using this result, we shall give some applications to circle action and finite cyclic group action on unitary manifolds and smooth manifolds. The rest of this section is to introduce and prove this algebraic result and the next two sections are devoted to applications.

Let $F$ be a field (finite or infinite). What we are mainly concerned with are the real number filed $\mathbb{R}$ and finite field $\mathbb{Z} / p \mathbb{Z}=: \mathbb{Z}_{p}$. Here $\mathbb{Z}$ is the integer ring and $p$ is a prime.

Suppose we have a set of $r$ elements $\left\{P_{1}, \cdots, P_{r}\right\}$. We call such a set a weighted set over $F$ if, for each $P_{i}, 1 \leq i \leq r$, we associate $n+1$ numbers $\mu_{i}, a_{1}^{(i)}, \cdots, a_{n}^{(i)}$ in $F$ to it. $\mu_{i}$ is called the coefficient of $P_{i}$ and $a_{1}^{(i)}, \cdots, a_{n}^{(i)}$ are called characteristic numbers of $P_{i}$. We will see in the next section that such a system appears naturally as the fixed points of group action on manifolds and the coefficients and characteristic numbers of these elements will be induced from the representation on the tangent spaces of the corresponding fixed points.

Now we will introduce the concept of stable weighted set.

Let $\lambda=\left(1^{m_{1}(\lambda)} 2^{m_{2}(\lambda)} \cdots n^{m_{n}(\lambda)}\right)$ be a partition of weight $w$, i.e., $m_{j}(\lambda)$ are all non-negative integers and $\sum_{j=1}^{n} j \cdot m_{j}(\lambda)=w$. For convenience we set

$$
a_{\lambda}^{(i)}:=\prod_{j=1}^{n}\left(a_{j}^{(i)}\right)^{m_{j}(\lambda)} .
$$

We call a weighted set $\left\{P_{1}, \cdots, P_{r}\right\}$ over $F$ stable if for any $0 \leq w<n$ and any partition $\lambda=\left(1^{m_{1}(\lambda)} 2^{m_{2}(\lambda)} \cdots n^{m_{n}(\lambda)}\right)$ of weight $w$, we have

$$
\Gamma(\lambda):=\sum_{i=1}^{r} \mu_{i} \cdot a_{\lambda}^{(i)}=0 .
$$

The reason of this definition lies in the localization formulae of group actions and its underlying geometric meaning will be clear in the next section.

\footnotetext{
*Received November 11, 2011; accepted for publication June 8, 2012.

${ }^{\dagger}$ Department of Mathematics, Tongji University, Shanghai 200092, China (pingli@tongji.edu.cn).

$\ddagger$ Department of Mathematics, University of California at Los Angeles, Los Angeles, CA 90095, USA; Center of Mathematical Science, Zhejiang University, Hangzhou 310027, China (liu@math.ucla.edu).
} 
For a partition $\lambda=\left(1^{m_{1}(\lambda)} 2^{m_{2}(\lambda)} \cdots n^{m_{n}(\lambda)}\right)$, we define

$$
m(\lambda):=\max \left\{m_{1}(\lambda), m_{2}(\lambda), \ldots, m_{n}(\lambda)\right\}
$$

With these notations understood, we can state the main result in this section.

Proposition 1.1. Let $\left\{P_{1}, \cdots, P_{r}\right\}$ be a stable weighted set over a field $F$. If there exists a partition $\lambda=\left(1^{m_{1}(\lambda)} 2^{m_{2}(\lambda)} \cdots n^{m_{n}(\lambda)}\right)$ of weight $n$ such that $m(\lambda) \geq r$, then

$$
\Gamma(\lambda)=\sum_{i=1}^{r} \mu_{i} \cdot a_{\lambda}^{(i)}=0
$$

Proof. Without loss of generality, we may assume $m_{1}(\lambda)=m(\lambda)$. Let

$$
\left\{a_{1}^{(i)} \mid 1 \leq i \leq r\right\}=\left\{s_{1}, \ldots, s_{l}\right\} \subset F
$$

By definition $s_{1}, \ldots, s_{l}$ are mutually distinct, $l \leq r$, and $l=r$ if and only if $a_{1}^{(i)}, \ldots, a_{n}^{(i)}$ are mutually distinct. Define

$$
A_{t}:=\sum_{\substack{1 \leq i \leq r \\ a_{1}^{(i)}=s_{t}}} \mu_{i} \cdot\left(a_{2}^{(i)}\right)^{m_{2}(\lambda)}\left(a_{3}^{(i)}\right)^{m_{3}(\lambda)} \cdots\left(a_{n}^{(i)}\right)^{m_{n}(\lambda)}, \quad 1 \leq t \leq l .
$$

Now let us consider the following $m_{1}(\lambda)$ partitions:

$$
\lambda^{(j)}:=\left(1^{j} 2^{m_{2}(\lambda)} 3^{m_{3}(\lambda)} \cdots n^{m_{n}(\lambda)}\right), \quad 0 \leq j \leq m_{1}(\lambda)-1 .
$$

The weights of these $\lambda^{(j)}$ are all less than $n$ as the weight of $\lambda$ is exactly $n$. Then following (1.1) we have

$$
\left\{\begin{array}{l}
A_{1}+A_{2}+\cdots+A_{l}=0 \\
s_{1} A_{1}+s_{2} A_{2}+\cdots+s_{l} A_{l}=0 \\
\vdots \\
\left(s_{1}\right)^{m_{1}(\lambda)-1} A_{1}+\left(s_{2}\right)^{m_{1}(\lambda)-1} A_{2}+\cdots+\left(s_{l}\right)^{m_{1}(\lambda)-1} A_{l}=0 .
\end{array}\right.
$$

Note that $l \leq r$ and by assumption $m_{1}(\lambda)=m(\lambda) \geq r$, which follows $l \leq m_{1}(\lambda)$. By definition $s_{1}, \cdots, s_{l}$ are mutually distinct, which means the determinant of the coefficient matrix of the first $l$ lines of (1.3) is

$$
\left|\begin{array}{cccc}
1 & 1 & \cdots & 1 \\
s_{1} & s_{2} & \cdots & s_{l} \\
\vdots & \vdots & \ddots & \vdots \\
\left(s_{1}\right)^{l-1} & \left(s_{2}\right)^{l-1} & \cdots & \left(s_{l}\right)^{l-1}
\end{array}\right|=\prod_{1 \leq i<j \leq l}\left(s_{j}-s_{i}\right) \neq 0
$$

which is a nonsingular Vandermonde matrix.

Thus

$$
A_{1}=A_{2}=\cdots=A_{l}=0 .
$$


Therefore,

$$
\begin{aligned}
\Gamma(\lambda) & =\sum_{i=1}^{r} \mu_{i} \cdot a_{\lambda}^{(i)} \\
& =\sum_{i=1}^{r}\left(a_{1}^{(i)}\right)^{m_{1}(\lambda)} \cdot \mu_{i} \cdot\left(a_{2}^{(i)}\right)^{m_{2}(\lambda)} \cdots\left(a_{n}^{(i)}\right)^{m_{n}(\lambda)} \\
& =\sum_{t=1}^{l}\left(s_{t}\right)^{m_{1}(\lambda)} \cdot A_{t}=0
\end{aligned}
$$

Remark 1.2. The idea of this proof can be traced back to Pelayo-Tolman ([8], Lemma 8) and the present authors (([6], Lemmas 3.1 and 3.2), ([5], Lemmas 3.2 and $3.2)$ ). Lü-Tan also used this idea in their proof of Theorem 1.1 in [7], which was extracted by Cho-Kim-Park in ([2], Theorem 2.2) without pointing it out explicitly.

Corollary 1.3. Let $\left\{P_{1}, \cdots, P_{r}\right\}$ be a stable weighted set over a field $F$. If we define

$$
m:=\max \{m(\lambda) \mid \lambda: \text { partitions of weight } n \text { such that } \Gamma(\lambda) \neq 0\}
$$

then

$$
r \geq m+1
$$

Note that if in Proposition $1.1 F$ is a finite field, then by (1.2) we know $l \leq|F|$, where $|F|$ is the cardinality of $F$. Therefore we have the following implication from the process of proving Proposition 1.1.

Corollary 1.4. Let $\left\{P_{1}, \cdots, P_{r}\right\}$ be a stable weighted set over a finite field $F$. If there exists a partition $\lambda=\left(1^{m_{1}(\lambda)} 2^{m_{2}(\lambda)} \cdots n^{m_{n}(\lambda)}\right)$ of weight $n$ such that $m(\lambda) \geq|F|$, then $\Gamma(\lambda)=0$.

Corollary 1.5. Let $\left\{P_{1}, \cdots, P_{r}\right\}$ be a stable weighted set over a finite field $F$. If we define

$$
m:=\max \{m(\lambda) \mid \lambda: \text { partitions of weight } n \text { such that } \Gamma(\lambda) \neq 0\},
$$

then

$$
|F| \geq m+1
$$

2. Applications to circle actions. For our purpose, here we only review Bott residue formula for $4 n$-dimensional smooth manifolds. For general case we refer to ([6], Section 2).

Let $N^{4 n}$ be a $4 n$-dimensional connected, oriented smooth manifold with a (smooth) circle action whose fixed points are non-empty and isolated, say 
$\left\{P_{1}, P_{2} \cdots, P_{r}\right\}$. At each fixed point $P_{i}$, the tangent space $T_{P_{i}} N$ splits as a real $S^{1}$-module induced from the isotropy representation as follows

$$
T_{p_{i}} N=\bigoplus_{j=1}^{2 n} V_{j}^{(i)}
$$

where each $V_{j}^{(i)}$ is a real 2-dimensional plane. We choose an isomorphism of complex plane $\mathbb{C}$ with $V_{j}^{(i)}$ relative to which the representation of $S^{1}$ on $V_{j}^{(i)}$ is given by $e^{\sqrt{-1} \theta} \mapsto e^{\sqrt{-1} k_{j}^{(i)} \theta}$ with $k_{j}^{(i)} \in \mathbb{Z}-\{0\}$. We can assume the rotation numbers $k_{1}^{(i)}, \cdots, k_{2 n}^{(i)}$ be chosen in such a way that the usual orientations on the summands $V_{j}^{(i)} \cong \mathbb{C}$ induce the given orientation on $T_{p_{i}} N$. Note that these $k_{1}^{(i)}, \cdots, k_{2 n}^{(i)}$ are uniquely defined up to even number of sign changes. In particular, their product $\prod_{j=1}^{2 n} k_{j}^{(i)}$ is well-defined.

Let $p_{j} \in H^{4 j}(M ; \mathbb{Z})(1 \leq j \leq n)$ be the $j$-th Pontrjagin class of $N^{4 n}$ and $\lambda=\left(1^{m_{1}(\lambda)} 2^{m_{2}(\lambda)} \cdots n^{m_{n}(\lambda)}\right)$ be a partition. Then we can define the corresponding Pontrjagin number $p_{\lambda}[N]$ as follows

$$
p_{\lambda}[N]:=<p_{1}^{m_{1}(\lambda)} p_{2}^{m_{2}(\lambda)} \cdots p_{n}^{m_{n}(\lambda)},[N]>.
$$

Here $[N]$ is the fundamental class of $N^{4 n}$ determined by the orientation and $\langle\cdot, \cdot\rangle$ is the Kronecker pairing. By definition $p_{\lambda}[N]=0$ unless the weight of $\lambda$ is $n$.

Let $e_{j}\left(x_{1}, \cdots, x_{2 n}\right)$ be the $j$-th elementary symmetric polynomial in the variables $x_{1}, \cdots, x_{2 n}$. At each fixed point $P_{i}$, we can associate to $n+1$ numbers $\mu_{i}, a_{1}^{(i)}, \cdots, a_{n}^{(i)}$ as follows.

$$
\mu_{i}=\frac{1}{\prod_{j=1}^{2 n} k_{j}^{(i)}}, \quad a_{j}^{(i)}=e_{j}\left(\left(k_{1}^{(i)}\right)^{2}, \cdots,\left(k_{2 n}^{(i)}\right)^{2}\right), \quad 1 \leq j \leq n .
$$

The following result is a special case of Bott residue formula ([1], p.598).

THEOREM 2.1 (Bott residue formula). With all above notations understood and suppose $\lambda=\left(1^{m_{1}(\lambda)} 2^{m_{2}(\lambda)} \cdots n^{m_{n}(\lambda)}\right)$ is any partition whose weight is no more than $n$, then we have

$$
\sum_{i=1}^{r} \mu_{i} \cdot a_{\lambda}^{(i)}=p_{\lambda}[N]
$$

Combining this formula with Proposition 1.1 will lead to the following result, which is parallel to ([2], Theorem 1.3) and a generalization of ([6], Theorem 1.4) in the smooth case.

THEOREM 2.2. Suppose $N^{4 n}$ is a $4 n$-dimensional connected, closed and oriented smooth manifold and there exists a partition $\lambda=\left(1^{m_{1}(\lambda)} 2^{m_{2}(\lambda)} \cdots n^{m_{n}(\lambda)}\right)$ of weight $n$ such that $p_{\lambda}[N] \neq 0$, then any circle action on $N^{4 n}$ has at least $m(\lambda)+1$ fixed points.

REMARK 2.3. In the statement of above theorem, we require no restriction that the circle action must have non-empty isolated fixed points. The reason is as follows. The non-vanishing of some Pontrjagin number guarantees that the fixed points of any circle action must be non-empty. If the fixed-point set is not isolated, then at least 
one connected component of it is a submanifold of positive dimension and in this case there are infinitely many fixed points.

Corollary 2.4. Suppose $N^{4 n}$ is a $4 n$-dimensional connected, closed and oriented smooth manifold. Let

$$
m:=\max \left\{m(\lambda) \mid p_{\lambda}[N] \neq 0\right\} .
$$

Then any circle action on $N^{4 n}$ has at least $m+1$ fixed points.

3. Applications to $\mathbb{Z}_{p}$ actions. Let us recall the notation of unitary manifold (some people call it stably almost-complex manifold or weakly almost-complex manifold), which is a generalization of that of almost-complex manifold.

Let $M^{2 n}$ be a $2 n$-dimensional connected, closed and smooth manifold. $M^{2 n}$ is called a unitary manifold if it is endowed with a complex vector bundle structure on the stable tangent bundle. More precisely, we can give a complex vector bundle structure on $T M \oplus \theta^{2 l}$, where $T M$ is the tangent bundle of $M$ and $\theta^{2 l}$ is the product

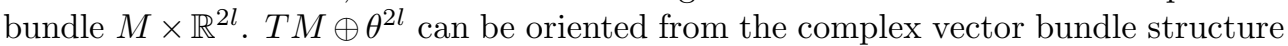
and $\theta^{2 l}$ is also oriented in the usual way. These orientations induce an orientation of $T M$. Hereafter a unitary manifold $M^{2 n}$ is always oriented in such a way.

Now suppose the unitary manifold $M^{2 n}$ admits a $\mathbb{Z}_{p}$ action preserving the given complex vector bundle structure. Here of course $p$ is a prime and $\mathbb{Z}_{p}$ is the cyclic group of order $p$. Thus $\mathbb{Z}_{p}$ is a finite field. Moreover we suppose this action has isolated fixed points, say $P_{1}, \cdots, P_{r}$. Then at each fixed point $P_{i}, \mathbb{Z}_{p}$ acts linearly on the complex vector space $V_{i}:=T_{P_{i}} M \oplus \mathbb{R}^{2 l}$ and the complex subspace of fixed vectors of this action is exactly $\mathbb{R}^{2 l}$. Thus $V_{i} / \mathbb{R}^{2 l}$ is a complex $\mathbb{Z}_{p}$-module without trivial factor, which is isomorphic to $T_{P_{i}} M$ as real vector space. So we have $n$ weights $k_{1}^{(i)}, \cdots, k_{n}^{(i)} \in \mathbb{Z}_{p}-\{0\}$ induced from this $\mathbb{Z}_{p}$-representation.

Note that, at each $P_{i}$, the tangent space $T_{P_{i}} M$ has two orientations. One is induced from that of $M$ and the other is induced from the complex structure of $V_{i} / \mathbb{R}^{2 l}$. We set $\epsilon\left(P_{i}\right)=+1$ or -1 according to these two orientations coincide or not. Clearly $\epsilon\left(P_{i}\right)=+1$ if $M^{2 n}$ is an almost-complex manifold.

Let $c_{j} \in H^{2 j}(M ; \mathbb{Z})(1 \leq j \leq n)$ be the $j$-th Chern class of the complex vector bundle $T M \oplus \theta^{2 l}$. Given any partition $\lambda=\left(1^{m_{1}(\lambda)} 2^{m_{2}(\lambda)} \cdots n^{m_{n}(\lambda)}\right)$, we can define the corresponding Chern number $c_{\lambda}[M]$ :

$$
c_{\lambda}[M]:=<c_{1}^{m_{1}(\lambda)} c_{2}^{m_{2}(\lambda)} \cdots c_{n}^{m_{n}(\lambda)},[M]>.
$$

By definition $c_{\lambda}[M]=0$ unless the weight of $\lambda$ is $n$.

At each fixed point $P_{i}(1 \leq i \leq r)$, we can associate to $n+1$ numbers $\mu_{i}, a_{1}^{(i)}, \cdots, a_{n}^{(i)} \in \mathbb{Z}_{p}$ as follows.

$$
\mu_{i}=\epsilon\left(P_{i}\right) \cdot\left(\prod_{j=1}^{n} k_{j}^{(i)}\right)^{-1}, \quad a_{j}^{(i)}=e_{j}\left(k_{1}^{(i)}, \cdots, k_{n}^{(i)}\right), \quad 1 \leq j \leq n .
$$

Here $e_{j}\left(x_{1}, \cdots, x_{n}\right)$ is the $j$-th elementary symmetric polynomial in the variables $x_{1}, \cdots, x_{n}$.

The following proposition is a special case of a result of Kosniowski ([4], Theorem 1.1), which reduces the calculations of the module $p$ Chern numbers of $M$ to the fixed points $\left\{P_{i}\right\}$ and is a $\mathbb{Z}_{p}$ analogue to Bott residue formula in the circle action. 
Proposition 3.1 (Kosniowski). With all these above understood and let $\lambda=$ $\left(1^{m_{1}(\lambda)} 2^{m_{2}(\lambda)} \cdots n^{m_{n}(\lambda)}\right)$ be any partition whose weight is no more than $n$. Then we have

$$
\sum_{i=1}^{r} \mu_{i} \cdot a_{\lambda}^{(i)} \equiv c_{\lambda}[M], \quad \bmod p
$$

Combining this proposition with Proposition 1.1 will lead to the following result.

THEOREM 3.2. Given a 2n-dimensional connected closed unitary manifold $M^{2 n}$ and a prime $p$. If there exists a partition $\lambda=\left(1^{m_{1}(\lambda)} 2^{m_{2}(\lambda)} \cdots n^{m_{n}(\lambda)}\right)$ of weight $n$ such that the corresponding Chern number $c_{\lambda}[M]$ is not divisible by $p$, then any $\mathbb{Z}_{p}$ action on $M^{2 n}$ has at least $m(\lambda)+1$ fixed points.

REMARK 3.3. Here we still don't need the assumption that the $\mathbb{Z}_{p}$ action has isolated fixed points and the reason is the same as that of Remark 2.3.

Corollary 3.4. Given a $2 n$-dimensional connected closed unitary manifold $M^{2 n}$ and a prime $p$. Let

$$
m:=\max \left\{m(\lambda) \mid c_{\lambda}[M] \text { is not divisible by } p\right\}
$$

Then any $\mathbb{Z}_{p}$ action on $M^{2 n}$ has at least $m+1$ fixed points.

The following result is an obstruction to the existence of a $\mathbb{Z}_{p}$ action on $M^{2 n}$ with isolated fixed points, which is a direct application of Corollary 1.5.

THEOREM 3.5. Given a 2n-dimensional connected closed unitary manifold $M^{2 n}$ and a prime $p$. Let

$$
m:=\max \left\{m(\lambda) \mid c_{\lambda}[M] \text { is not divisible by } p\right\}
$$

If there exists a $\mathbb{Z}_{p}$ action on $M^{2 n}$ with isolated fixed points, then

$$
p \geq m+1
$$

REMARK 3.6.

1. As mentioned in ([4], p. 284), there is a similar formula like Proposition 3.1 in the smooth case for $\mathbb{Z}_{p}$ actions so long as $p$ is an odd prime. So we have similar results like Theorem 3.2, Corollary 3.4 and Theorem 3.5 for smooth $\mathbb{Z}_{p}$ action on $4 n$-dimensional smooth, closed and oriented manifolds in terms of Pontrjagin numbers so long as $p$ is an odd prime.

2. If $p$ is an odd prime and $\mathbb{Z}_{p}$ acts smoothly on a closed, oriented, smooth manifold $N^{2 n}$ with isolated fixed points such that the fixed points satisfy some additional assumption, then Ewing and Kosniowski also gives a lower bound of the number of these fixed points in terms of $n$ and $p$ ([3], p. 295). 
Acknowledgments. The first author was partially supported by National Natural Science Foundation of China (Grant No. 11101308) and JSPS Postdoctoral Fellowship for Foreign Researchers.

\section{REFERENCES}

[1] M. F. Atiyah and I. M. Singer, The index theory of elliptic operators: III, Ann. Math., 87 (1968), pp. 546-604.

[2] H. Cho, J. Kim, And H. Park, On the conjecture of Kosniowski, Asian. J. Math., 16 (2012), pp. $271-278$.

[3] J. EwIng, C. Kosniowski, The number of fixed points of cyclic group actions, Bull. London Math. Soc., 16 (1984), pp. 295-302.

[4] C. Kosniowski, Characteristic numbers of $\mathbb{Z} / p$ manifolds, J. London Math. Soc., 14 (1976), pp. 283-295.

[5] P. LI, Circle action, lower bound of fixed points and characteristic numbers, J. Fixed Point Theory Appl., 11 (2012), pp. 245-251.

[6] P. Li AND K. Liu, Some remarks on circle action on manifolds, Math. Res. Letters., 18 (2011), pp. 437-446.

7] Z. L ̈̈ AND Q. TAN, Equivariant Chern numbers and the number of fixed points for unitary torus manifolds, Math. Res. Letters., 18 (2011), pp. 1319-1325.

[8] A. Pelayo and S. Tolman, Fixed points of symplectic periodic flows, Ergodic Theory Dynam. Systems, 31 (2011), pp. 1237-1247. 
P. LI AND K. LIU 